\title{
Study on the Experimental Teaching for Undergraduates in Requirements of the Plan for Educating and Training Excellent Engineers
}

\author{
X.D. Yuan \\ Materials Science and Engineering Institute \\ Shandong Jianzhu University \\ Jinan, China
}

\author{
X.J. Yang \\ Shandong Product Quality Inspection Research Institute \\ Ji'nan, 250100, China \\ Shandong Key Laboratory for Testing Technology of Material \\ Chemical Safety \\ Ji’nan, 250103, China
}

\author{
G.L. Yuan \\ Jinan Engineering Quality and Work Safety Supervision Station \\ Ji’nan, 250013, China
}

\begin{abstract}
In requirements of the plan for educating and training outstanding engineers, the experimental resources were integrated by School of materials science and engineering, Shandong Jianzhu University, the opening experimental teaching system was founded, and the experiment technical skills of students were improved, especially metallographic skills. The influence of the opening experimental teaching system on the experiment technical ability of undergraduates was studied taking the study on the microstructure of Cr-Rare-boronized layer under low temperatures as an example, and reasons were also analyzed.
\end{abstract}

Keywords-experiment teaching; outstanding engineers; Cr-rare earth-boronizing; steel 45

\section{INTRODUCTION}

The plan for educating and training outstanding engineers (referred to as "outstanding program"), is a major reform project implementing the "national medium and long-term educational reform and development plan (2010-2020)" and "national long-term talent development planning outline (2010-2020)". The universities and colleges formulate training goals of the engineering and technical personnel of the school in accordance with the industry standard requirements and combined with the school characteristic and the talent training standards under the guidance of general standards, since the beginning of June 23, 2010. In the "outstanding program" requirements, studies, which include the teaching reform of school and professional, personnel training mode, school enterprise cooperation and the reform of teaching practice were carried out by some universities and colleges ${ }^{[1-7]}$.

Shandong Jianzhu University is a provincial university giving priority to engineering, with balanced development of engineering, science, management, economy, arts and law. Over the past years, applied talents with the ability of engineering practice were cultivated. Material forming and control engineering in school of materials science and engineering is approved as the 2013 third batch of "outstanding program", which was organized by the ministry of education. In the requirements of "outstanding program", studies about the demand for technical personnel materials engineering in the new period was carried out combing the characteristics of the material science itself. The experimental resources were integrated, the opening experimental teaching system was founded, and the experiment technical skills of students were improved, especially metallographic skills.

In this paper, the influence of the opening experimental teaching system on the experiment technical ability of undergraduates was studied taking the study on the microstructure of Cr-Rare-boronized layer under low temperatures as an example, especially metallographic skills, and reasons were also analyzed.

\section{IMPLEMENTATION OF THE OPENING EXPERIMENT TEACHING SYSTEM AND IMPROVEMENT OF THE ABILITY TO SOLVE PROBLEMS}

The opening experiment teaching system was implemented and the duration was one month. Students design the experiment independently, operate the equipment independently, and deal with the experimental data independently.

Students design the experiment scheme according to practical problems encountered in the enterprise, apply for the new experiment project by themselves (they may receive the money, 2500 Yuan, from the school). Practical engineering problems can be solved through the experimental study. Experimental results can also be used to participate in various national university science and technology competition, from which the students' employment competitiveness can be improved. Each year the University subsidized student autonomous application topic more than 30 items, a total 
funding of research funds was more than 100000 Yuan. The ability of analyzing the question, solving the problem, and hands-on activities was enhanced through the implementation of opening experiment teaching system. The specific experimental teaching mode was shown in figure 1 .

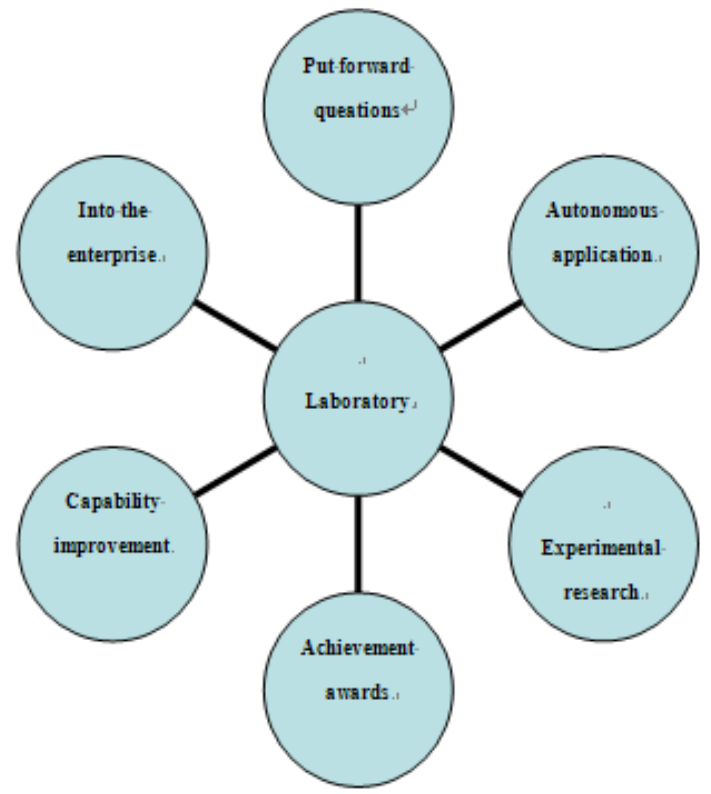

FIGURE I. MODE OF OPENING EXPERIMENT TEACHING SYSTEM.

\section{IMPROVEMENT OF THE ABILITY OF METALLOGRAPHIC SAMPLE REPARATION}

\section{A. Experiment Process}

The main material for the experiment is steel 45 . The samples were first quenched in water at $860^{\circ} \mathrm{C}$ for $30 \mathrm{~min}$. The Cr-Rare earth-boronizing process was performed under $600^{\circ} \mathrm{C}$ $\times 6 \mathrm{~h}$ on the surface of the quenched samples. The boronized samples were then set on an inlaying machine, polished using electronic grinder until the specimen surface was smooth. Then samples were grinded by using 200\# sandpaper gently, and the grinding direction can not be changed for 3 minutes so that the specimen surface further can be bright. The samples were grinded on sandpaper immediately, which was coated with white cat detergent on the surface. The general milling time was 5 minutes. Then samples were polished, cleaned, and dried. Metallographic observation was performed on an optical microscopy.

\section{B. Microstructure of the Cr-Rare-boronized Layer}

The microstructure of the boride layer was shown in Figure 2. It can be seen that the boride layer is integrity, continuous, and uniform.

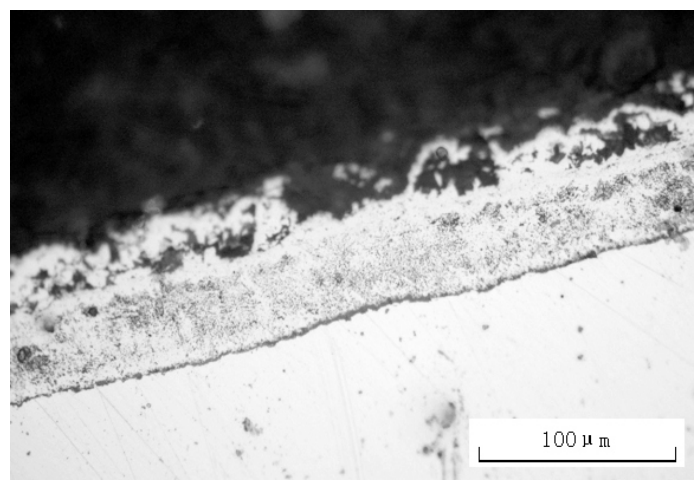

FIGURE II. MICROSTRUCTURE OF THE BORIDE LAYER.

Parts of boride layer shedded during the process of grinder grinding due to its higher hardness and brittleness. Black holes and micro cracks were observed. The shedding phenomenon continues and the microstructure was damaged during the sandpaper grinding. Those problems can not be solved by traditional grinding methods. The agent which contains a surface active agent was used to solve the above problems. The surface active agents play a major role in all kinds of detergent materials, which are sometimes referred to as the "active ingredients" or "effective". It is capable of reducing solvent surface tension of material at low concentrations. The dirt was washed off due to the existence of surfactants in detergent, which lead to the wetting emulsification, dispersion and produce, foaming, and solubilization. The hard particles produced in the process of sandpaper grinding will be removed under the action of surfactant, and does not exist between the surface of the sp0ecimen and sandpaper acting as a "second phase particles". The boride layer will not fall off.

The presence of sodium silicate in detergents, which was known as the "water glass" and "sodium silicate" plays an important role in regulating the $\mathrm{PH}$ of the detergent and maintaining the detergent solution to certain alkalinity. The existence of sodium carbonate in detergent can improve the ability of removing oil. The detergent itself is alkaline due to the above substances, which will corroded the materials and reduce the later corrosion time. Furthermore, the corroded materials will be removed effectively and the damage degree of boride layer will be reduced.

\section{CONCLUSIONS}

Opening experiment teaching system was implemented by school of materials science and engineering of Shandong Jianzhu University after the "outstanding program" was approved. The three-dimensional platform between the laboratory, businesses and industries was used, and the ability of metallographic sample reparation was improved comprehensively.

\section{ACKNOWLEDGEMENT}

Sponsored by the Research Fund for the College science and technology plan of Shandong Province ( Project No. J12LA11).

\section{REFERENCE}

[1] Sun Z Z, Chen S G, Zhong S Y. Training mode of enterprise engineering 
practice for outstanding engineers [J].Research and exploration in laboratory, 2012, 31(8):285-289.

[2] Chen S. Reform of practice teaching according to mode of applied cultivation [J]. Research and exploration in laboratory, 2012, 31(8):1-4.

[3] Zhao J H. Review of studies on the effectiveness of undergraduate experimental teaching in the west [J]. Research and exploration in laboratory, 2012, 31(10):91-94.

[4] Zhang X M. Innovative research and practice on "project supermarket" mode of opening experiment. [J]. Experimental technology and management, 2012, 29(10):33-36.

[5] Chen N N. Opening laboratory in engineering colleges and universities and reform of experiment teaching. [J]. Experimental technology and management, 2006, 23(4):110-112.

[6] Lai Z H. On experimental teaching reform and practice of civil engineering major [J]. Research and exploration in laboratory, 2010 29(11):319-321.

[7] Wang H Q, Wu Q F, Liang X J. Discussion on reform of university physics experiments by taking application as guidance. [J]. Experimental technology and management, 2012, 29(9):148-150. 